\title{
Serum uric acid as a predictor of mortality and future exacerbations of COPD
}

\author{
Konstantinos Bartziokas ${ }^{1,4}$, Andriana I. Papaioannou²,4, Stelios Loukides ${ }^{3}$, \\ Alexandros Papadopoulos ${ }^{1}$, Aikaterini Haniotou', Spyridon Papiris ${ }^{3}$ and \\ Konstantinos Kostikas ${ }^{3}$ \\ Affiliations: \\ ${ }^{1}$ Respiratory Medicine Dept, Amalia Fleming General Hospital, Athens, \\ ${ }^{2} 3 r d$ Respiratory Medicine Dept, Sismanogleion General Hospital, Athens, and \\ ${ }^{3}$ 2nd Respiratory Medicine Dept, University of Athens Medical School, Athens, Greece. \\ ${ }^{4}$ Both authors contributed equally.
}

Correspondence: K. Kostikas, 2nd Respiratory Medicine Dept, University of Athens Medical School, Stamouli 3 , Karditsa 43100, Greece. E-mail: ktkdotenet.gr

ABSTRACT Serum uric acid is increased in respiratory disease, especially in the presence of hypoxia and systemic inflammation. We evaluated serum uric acid as a biomarker for prediction of mortality and future acute exacerbation of chronic obstructive pulmonary disease (AECOPD).

Serum uric acid was measured in 314 eligible consecutive patients on admission for AECOPD. Patients were evaluated monthly for 1 year.

Uric acid levels were higher in patients with more severe airflow limitation and in those experiencing frequent exacerbations. High uric acid levels $\left(\geqslant 6.9 \mathrm{mg} \cdot \mathrm{dL}^{-1}\right)$ were an independent predictor of 30 -day mortality in multivariate Cox regression analysis (HR 1.317, 95\% CI 1.011-1.736; p=0.044), but not of 1-year mortality. Patients with high serum uric acid required more prolonged hospitalisation, and more often needed noninvasive ventilation and admission to the intensive care unit within 30 days. In addition, high uric acid levels were associated with increased risk and hospitalisation for AECOPD in 1 year in multivariate Poisson regression analysis (incidence rate ratio 1.184 (95\% CI 1.125-1.246) and 1.190 (95\% CI 1.105-1.282), respectively; both $\mathrm{p}<0.001$ ).

Serum uric acid is associated with increased 30-day mortality and risk for AECOPD and hospitalisations in 1-year follow-up. This low-cost biomarker may be useful in the identification of high-risk chronic obstructive pulmonary disease patients that could benefit from intensive management.

@ERSpublications

Serum uric acid was linked with airflow limitation in COPD and predicted mortality and future exacerbations http://ow.ly/qflaZ 


\section{Introduction}

Chronic obstructive pulmonary disease (COPD) is one of the leading causes of morbidity and mortality with increasing prevalence worldwide [1]. An acute exacerbation of COPD (AECOPD) is characterised by a significant change in symptoms that is acute in onset and may warrant a change in regular medication [1]. Some COPD patients are particularly susceptible to exacerbations, and this has a negative impact on survival [2].

Serum uric acid is the final product of purine degradation [3], which increases significantly during hypoxia [4]. Elevated uric acid levels have been associated with the presence of systemic inflammation [5] and increased cardiovascular risk [6]. In this context, increased levels of uric acid have been shown in respiratory disorders, including obstructive sleep apnoea [7] and pulmonary hypertension [8]. In COPD, cigarette smoke induces oxidative stress and lung inflammation, resulting in lung tissue damage and decline of pulmonary function [1]. Impairment of pulmonary function reduces oxygen intake, resulting in tissue hypoxia which is more prominent during AECOPD. Serum uric acid levels have been associated with the presence of airflow obstruction in a general population in Japan [9], whereas a small cross-sectional study showed significant associations between serum uric acid to creatinine ratio and spirometry values and dyspnoea in COPD patients [10]. Despite the above evidence, to date, no study has evaluated the role of uric acid levels on admission for acute exacerbations of COPD on the outcome of exacerbation and the longterm survival and exacerbation frequency of COPD patients.

The aim of the present prospective cohort study was to evaluate the possible role of serum uric acid as a biomarker for the prediction of the outcome of patients hospitalised for AECOPD. The primary end-points were all-cause mortality at 30 days and 1 year. Secondary outcomes included the duration of hospitalisation, the time to the first AECOPD and hospitalisation for acute exacerbation of COPD, and the frequency of those events in 1-year follow-up. Finally, we evaluated associations of serum uric acid levels with clinically relevant parameters in COPD patients. Preliminary results from this study have been reported previously [11].

\section{Methods}

Patients

We enrolled consecutive patients admitted for AECOPD to the respiratory medicine department of the Amalia Fleming General Hospital (Athens, Greece) between November 2008 and December 2010. All subjects were current or ex-smokers ( $\geqslant 20$ pack-years) with a previous diagnosis of COPD by a respiratory physician. Patients with other acute respiratory conditions, a history of respiratory disorders other than COPD, inability or unwillingness to cooperate with the investigators, or without available spirometry data were excluded. In addition, we excluded patients with chronic comorbidities that significantly influence uric acid levels (i.e. chronic renal failure, severe liver dysfunction or malignancies). All patients were invited to participate in the present study on the first day of admission to the respiratory ward by a study investigator, who allowed them enough time to decide and assured them that their care would not be affected by their decision to participate in the study. The study protocol was approved by the local ethics committee and all participants provided written informed consent.

\section{Study design}

Patient demographics were recorded, including age, sex, body mass index (BMI), smoking habit, treatment prior to admission and comorbidities, with special emphasis on cardiovascular disease (hypertension, coronary artery disease, congestive heart failure, arrhythmias or stroke) and calculation of Charlson's comorbidity index score [12]. Clinical parameters were recorded on admission, including vital signs, level of dyspnoea (Borg and modified Medical Research Council (MRC) scales) and arterial blood gases ( $\mathrm{pH}$, arterial carbon dioxide tension, arterial oxygen tension $\left(\mathrm{PaO}_{2}\right)$ and arterial oxygen saturation). Blood samples were collected from each patient at the time of admission to the emergency department and prior to the initiation of any treatment for serum uric acid and standard laboratory measurements (complete blood count, serum creatinine and C-reactive protein (CRP)). Decisions on treatment and discharge were made by attending physicians not involved in this study, according to the Global Initiative for Chronic Obstructive Lung Disease (GOLD) guidelines [1]. The diagnosis and classification of airflow limitation was based on post-bronchodilator spirometry in a stable condition, i.e. in the previous 6 months and $\geqslant 4$ weeks prior to admission from the patients' records or 4-8 weeks after discharge, according to GOLD guidelines (patients were classified as GOLD stages 1-4) [1].

Patients were evaluated on admission and at discharge by the study investigators and were followed up for 1 year by monthly telephone interviews. In those interviews, a study investigator recorded the patients' vital status and the numbers of new AECOPD (defined as need for antibiotics and/or oral corticosteroids, visits 
to the emergency department and/or hospitalisations). The recording of hospitalisations for AECOPD was based on self-reporting of patients and was confirmed by hospital records where necessary. In the event that a patient could not be reached by a regular monthly communication, every effort was made to obtain all the necessary information on a following communication. Patients with two or more exacerbations per year were defined as frequent exacerbators [13].

\section{Study outcomes}

The primary end-points were all-cause mortality at 30 days and 1 year. Secondary outcomes included duration of hospitalisation, need for mechanical ventilation and time to the first AECOPD and hospitalisation for AECOPD, as well as the frequency of those events in 1 year.

\section{Statistical analysis}

Data are presented as $\mathrm{n}(\%)$ for categorical variables, mean \pm SD for normally distributed values and median (interquartile range (IQR)) for skewed numerical variables. Comparisons between groups were performed using Chi-squared tests and unpaired t-tests or Mann-Whitney U-tests. Correlations were performed with Spearman's rank correlation coefficient.

A power analysis was performed using the PS Power and Sample Size Calculation programme (http:// biostat.mc.vanderbilt.edu/PowerSampleSize) based on data from a previous study by Høiseth et al. [14]. Briefly, a minimum number of 60 participants would be required in the high uric acid group in order to achieve a type I error probability of 0.05 with $80 \%$ power for a relative mortality ratio of 0.58 between patients with high and low uric acid levels for a median follow-up of 1.9 years and a median survival of 50 months after hospitalisation for acute exacerbation of COPD [15].

For the analysis of the primary objective, survival analyses and Cox and Poisson regression analyses were implemented. In detail, the times to death, first AECOPD and hospitalisation for acute exacerbation of COPD according to the presence or absence of elevated serum uric acid levels were evaluated with KaplanMeier survival curves and log-rank tests. AECOPD and hospitalisations are both reported as the proportion of patients undergoing an event as well as the mean number of events per patient per year.

Receiver operating characteristics (ROC) curve analysis was performed for the evaluation of the performance of serum uric acid in the prediction of 30-day mortality. Cox regression univariate and multivariate analyses were performed in order to evaluate the influence of serum uric acid levels on 30-day and 1-year mortality. Significant confounders evaluated in Cox regression analyses included age, BMI, sex, current smoking status, Charlson score and GOLD stage. Results are presented as hazard ratios with $95 \%$ confidence intervals (CI). Poisson regression univariate and multivariate analyses were additionally performed in order to account for variability in exacerbation rates between patients [16]. Results are presented as incidence rate ratios (IRR) with 95\% CI. The previously mentioned significant confounders were additionally evaluated in the Poisson analysis and the results are presented as IRR with 95\% CI. Skewed data were logarithmically transformed for regression analyses. P-values $<0.05$ were considered statistically significant. Data were analysed using SPSS 17.0 for Windows (SPSS Inc., Chicago, IL, USA) and MedCalc 9 (MedCalc Software, Mariakerke, Belgium).

\section{Results}

We evaluated 426 consecutive patients admitted for AECOPD and 314 were eligible for inclusion in the present study. The flow chart of study participants is shown in figure 1. The demographic characteristics of the 314 patients who were included in data analysis are presented in table 1 . Patients were divided into two groups according to the presence of serum uric acid levels above or below the median value $\left(\geqslant 6.9 \mathrm{mg} \cdot \mathrm{dL}^{-1}\right.$ $\mathrm{n}=148$ or $<6.9 \mathrm{mg} \cdot \mathrm{dL}^{-1} \mathrm{n}=166$, respectively). Patients with high serum uric acid levels had more severe airflow obstruction, more comorbidity, more severe dyspnoea and more impaired oxygenation compared to patients with low serum uric acid levels.

Median (IQR) serum uric acid levels on admission were higher in patients with more severe airflow limitation (6.5 (6.2-6.8) $\mathrm{mg} \cdot \mathrm{dL}^{-1}, 6.6(6.3-6.9) \mathrm{mg} \cdot \mathrm{dL}^{-1}, 7.5(6.8-8.6) \mathrm{mg} \cdot \mathrm{dL}^{-1}$ and $8.6(8.1-9.7) \mathrm{mg} \cdot \mathrm{dL}^{-1}$ for GOLD stages $1-4$, respectively; $\mathrm{p}<0.001$ ) (fig. $2 \mathrm{a}$ ). Patients classified as GOLD stages 1 and 2 had significantly lower uric acid levels compared to patients in stages 3 and $4\left(6.6(6.3-6.9) \mathrm{mg} \cdot \mathrm{dL}^{-1}\right.$ and 7.8 $(7.0-8.9) \mathrm{mg} \cdot \mathrm{dL}^{-1}$, respectively; $\left.\mathrm{p}<0.001\right)$. Furthermore, uric acid levels were higher in frequent $(\mathrm{n}=170)$ compared to nonfrequent $(\mathrm{n}=144)$ exacerbators $\left(7.7(6.7-8.8) \mathrm{mg} \cdot \mathrm{dL}^{-1}\right.$ versus $6.6(6.3-6.9) \mathrm{mg} \cdot \mathrm{dL}^{-1}$, respectively; $\mathrm{p}<0.001$ ) (fig. $2 \mathrm{~b}$ ). Furthermore, patients with cardiovascular comorbidities had higher uric acid levels compared to those without cardiovascular comorbidities $\left(7.8(6.8-8.8) \mathrm{mg} \cdot \mathrm{dL}^{-1}\right.$ versus 6.6 (6.3-6.9) $\mathrm{mg} \cdot \mathrm{dL}^{-1}$, respectively; $\left.\mathrm{p}<0.001\right)$. 




FIGURE 1 Flow chart of the study participants. AECOPD: acute exacerbations of chronic obstructive pulmonary disease.

Serum uric acid levels and associations with clinically relevant outcomes

Associations of serum uric acid levels on admission with clinically relevant outcomes are presented in table 2. Serum uric acid levels presented positive correlations with Charlson comorbidity index score, Borg dyspnoea score, serum CRP and length of hospitalisation, as well as negative correlations with BMI, forced expiratory volume in $1 \mathrm{~s}(\mathrm{FEV} 1) \%$ predicted and $\mathrm{PaO}_{2}$ /inspiratory oxygen fraction $\left(\mathrm{FiO}_{2}\right)$ ratio. The scatterplot of serum uric acid and FEV1 \% pred is presented in figure $2 \mathrm{c}$.

A comparison of clinical outcomes at 30 days and 1 year in patients with low and high serum uric acid levels on admission is presented in table 3. Patients with high serum uric acid levels required more prolonged hospitalisation and presented higher rates of 30-day and 1-year mortality. Moreover, patients

\section{TABLE 1 Patient demographics}

\begin{tabular}{|c|c|c|c|c|}
\hline & All & $\begin{array}{l}\text { Low uric acid } \\
<6.9 \mathrm{mg} \cdot \mathrm{dL}^{-1}\end{array}$ & $\begin{array}{l}\text { High uric acid } \\
\geqslant 6.9 \mathrm{mg} \cdot \mathrm{dL}^{-1}\end{array}$ & p-value \\
\hline Subjects & 314 & 166 & 148 & \\
\hline Age years & $72(67-78)$ & $73(67-79)$ & $71(66-78)$ & 0.245 \\
\hline BMI $\mathrm{kg} \cdot \mathrm{m}^{-2}$ & $26.2(23.8-30.5)$ & $26.4(24.8-29.5)$ & $26.8(20.9-34.2)$ & 0.228 \\
\hline Current smokers & $83(26)$ & $46(28)$ & $37(25)$ & 0.610 \\
\hline \multicolumn{5}{|l|}{ GOLD stage } \\
\hline 1 & $39(12.4)$ & 37 (22.3) & $2(1.4)$ & \multirow[t]{4}{*}{$<0.001$} \\
\hline 2 & $117(37.3)$ & $90(54.2)$ & $27(18.2)$ & \\
\hline 3 & $119(37.9)$ & 37 (22.3) & $82(55.4)$ & \\
\hline 4 & $39(12.4)$ & $2(1.2)$ & $37(25)$ & \\
\hline Charlson score & $2.0(2.0-3.0)$ & $2.0(1.0-3.0)$ & $3.0(2.0-4.0)$ & 0.009 \\
\hline $\mathrm{PaCO}_{2} \mathrm{mmHg}$ & $40.4(35.0-55.1)$ & $40.1(32.9-49.5)$ & $32.9(36.0-57.3)$ & 0.020 \\
\hline CRP $\mathrm{mg} \cdot \mathrm{L}^{-1}$ & $3.8(1.0-8.7)$ & $3.2(0.7-5.4)$ & $5.0(1.9-12.5)$ & $<0.001$ \\
\hline AECOPD per patient per year & 2.45 & 1.53 & 3.47 & $<0.001$ \\
\hline Hospitalisations per patient per year & 1.07 & 0.57 & 1.65 & $<0.001$ \\
\hline
\end{tabular}

Data are presented as $\mathrm{n}, \mathrm{n}(\%)$ or median (interquartile range), unless otherwise stated. Bold indicates statistical significance. BMI: body mass index; FEV1: forced expiratory volume in $1 \mathrm{~s}$; GOLD: Global Initiative for Chronic Obstructive Lung Disease; $\mathrm{PaO}_{2}$ : arterial oxygen tension; FIO inspiratory oxygen fraction; $\mathrm{PaCO}_{2}$ : arterial carbon dioxide tension; CRP: C-reactive protein; AECOPD: acute exacerbation of chronic obstructive pulmonary disease. 

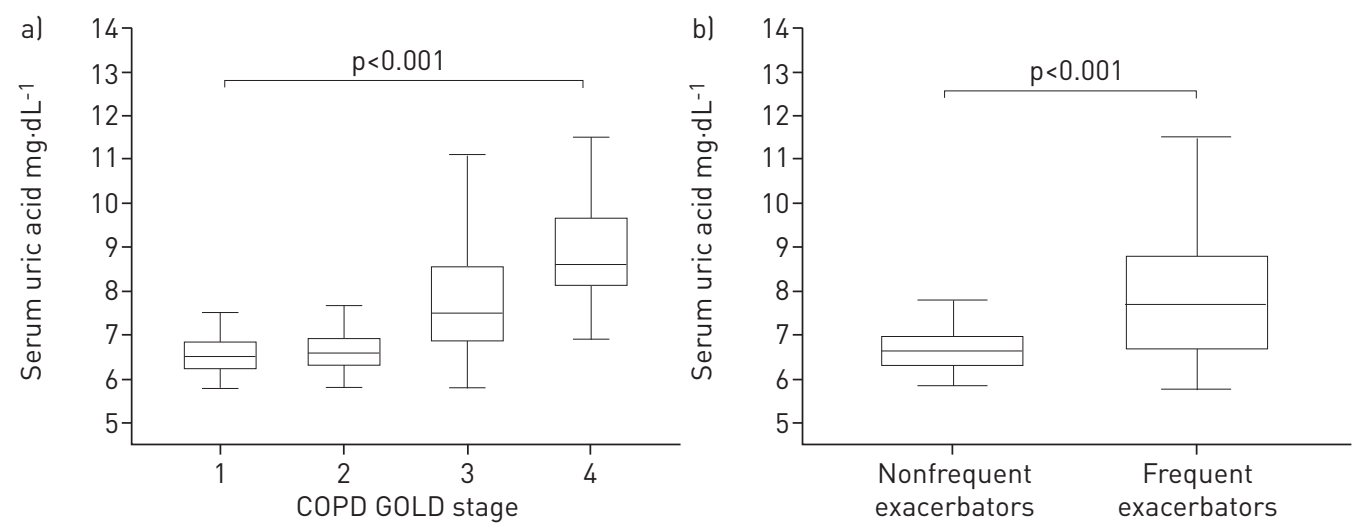

c) GOLD airflow obstruction severity

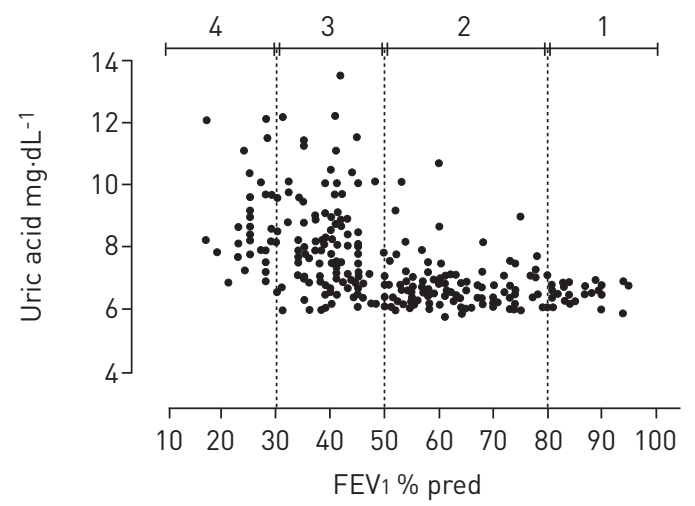

\begin{abstract}
FIGURE 2 Uric acid levels on admission in the study population according to a) airflow limitation severity (Global Initiative for Chronic Obstructive Lung Disease (GOLD) stage 1-4) and b) exacerbation frequency (nonfrequent $\left(0-1\right.$ events·year $\left.{ }^{-1}\right)$ versus frequent $\left(\geqslant 2\right.$ events.year $\left.{ }^{-1}\right)$ exacerbators.) c) Scatterplot of serum uric acid and forced expiratory volume in $1 \mathrm{~s}$ (FEV1). Correlation was performed with Spearman's rank correlation coefficient $\left(\mathrm{r}_{\mathrm{s}}=-0.599, \mathrm{p}<0.001\right)$. COPD: chronic obstructive pulmonary disease; \% pred: \% predicted.
\end{abstract}

with high serum uric acid levels required noninvasive ventilation (NIV) and intensive care unit (ICU) admission at 30 days more often, and had higher rates of acute exacerbations of COPD and hospitalisations in 1 year.

Serum uric acid levels and 30-day and 1-year mortality

In ROC analysis, serum uric acid presented an area under the curve of 0.738 (95\% CI 0.686-0.786) for the prediction of 30-day mortality, with a sensitivity of 0.81 and a specificity of 0.56 for the cut-off point of $6.9 \mathrm{mg} \cdot \mathrm{dL}^{-1}$ (fig. 3a). Kaplan-Meier survival curves evaluating time to death within 30 days according to serum uric acid levels are presented in figure 3b. Patients with high serum uric acid levels presented increased mortality risk at both 30 days and 1 year compared to those with low serum uric acid levels

\title{
TABLE 2 Associations of serum uric acid levels with clinically relevant outcomes
}

$r_{\mathrm{s}}$ coefficient

\begin{tabular}{lcc}
\hline Age years & -0.563 & 0.320 \\
Charlson score & 0.117 & 0.039 \\
$\mathrm{BMI} \mathbf{k g} \cdot \mathrm{m}^{-2}$ & -0.071 & 0.209 \\
$\mathrm{FEV} 1 \%$ predicted & -0.599 & $<0.001$ \\
Borg dyspnoea score & 0.332 & $<0.001$ \\
Arterial blood gases & & \\
$\mathrm{PaO}_{2} / \mathrm{FlO}_{2} \mathrm{mmHg}$ & -0.188 & 0.001 \\
$\mathrm{PaCO}_{2} \mathrm{mmHg}$ & 0.165 & 0.003 \\
Hospitalisation days & 0.630 & $<0.001$ \\
Serum CRP $\mathrm{mg}^{-1}$ & 0.287 & $<0.001$ \\
\hline
\end{tabular}

Bold indicates statistical significance. $r_{s}$ : Spearman's rank correlation coefficient; BMI: body mass index; FEV1: forced expiratory volume in $1 \mathrm{~s} ; \mathrm{PaO}_{2}$ : arterial oxygen tension; $\mathrm{FIO}_{2}$ : inspiratory oxygen fraction; $\mathrm{PaCO}_{2}$ : arterial carbon dioxide tension; CRP: C-reactive protein. 
TABLE 3 Comparison of clinical outcomes at 30 days and 1 year in patients with low and high serum uric acid levels

$\begin{array}{ccc}\begin{array}{c}\text { Low uric acid } \\ <6.9 \mathrm{mg} \cdot \mathbf{d L}^{-1}\end{array} & \begin{array}{c}\text { High uric acid } \\ \geqslant 6.9 \mathrm{mg} \cdot \mathrm{dL}^{-1}\end{array} & \text { p-value } \\ 166 & 148 & \\ 4.0(3.0-6.0) & 9.0(6.0-13.0) & <\mathbf{0 . 0 0 1} \\ 5(3) & 22(14.9) & <\mathbf{0 . 0 0 1} \\ 4(2.4) & 24(16.2) & <\mathbf{0 . 0 0 1} \\ 21(12.6) & 56(37.8) & <\mathbf{0 . 0 0 1} \\ 8(4.8) & 21(14.2) & \mathbf{0 . 0 0 4} \\ 1.0(0.0-2.0) & 3.0(2.0-5.0) & <\mathbf{0 . 0 0 1} \\ 0.0(0.0-1.0) & 1.0(0.0-3.0) & <\mathbf{0 . 0 0 1}\end{array}$

Data are presented as median (interquartile range) or $\mathrm{n}(\%)$, unless otherwise stated. Bold indicates statistical significance. ICU: intensive care unit; NIV: noninvasive ventilation; AECOPD: acute exacerbation of chronic obstructive pulmonary disease.

$(\mathrm{p}<0.001$ and $\mathrm{p}=0.004$, respectively, by log-rank test). In univariate Cox regression analyses, serum uric acid ratio was associated with increased risk of 30-day and 1-year mortality (table 4). However, in the multivariate analysis, the serum uric acid ratio remained an independent predictor of 30-day mortality (HR $1.317,95 \%$ CI 1.011-1.736; $\mathrm{p}=0.044)$, but was not an independent predictor of 1 -year mortality (HR 1.186 , $95 \%$ CI $0.925-1.521 ; \mathrm{p}=0.179)$.

Serum uric acid levels and AECOPD and hospitalisations at 1-year follow-up

Kaplan-Meier survival curves evaluating the time to first AECOPD and hospitalisation for AECOPD according to the levels of serum uric acid on admission are presented in figure $4(\mathrm{p}<0.001$ for both comparisons by log-rank test). In univariate Poisson regression analyses, serum uric acid levels were associated with a higher risk of AECOPD and hospitalisation for AECOPD (table 5). In the multivariate analyses, serum uric acid levels remained an independent predictor of a higher risk for AECOPD (IRR 1.184, 95\% CI 1.125-1.246; $\mathrm{p}<0.001$ ) and for hospitalisations for AECOPD (IRR 1.190, 95\% CI 1.105-1.282; $\mathrm{p}<0.001$ ) at 1 -year follow-up.

\section{Discussion}

In the present study we have shown that serum uric acid levels on admission for AECOPD were an independent predictor of 30-day mortality and were associated with a higher risk of AECOPD and hospitalisations for AECOPD at 1-year follow-up. Additionally, patients with increased uric acid levels required more prolonged hospitalisation, and more often required use of NIV and ICU admission at


FIGURE 3 a) Receiver operating characteristic curve evaluating the diagnostic performance of serum uric acid as a predictor of 30-day mortality. Area under the curve 0.738 ( $95 \%$ CI 0.686-0.786). b) Kaplan-Meier survival curves evaluating the time to death in days for patients with uric acid levels above $\left(\geqslant 6.9 \mathrm{mg} \cdot \mathrm{dL}^{-1}\right)$ and below $\left(<6.9 \mathrm{mg} \cdot \mathrm{dL}^{-1}\right)$ the median value $(\mathrm{p}<0.001$ by log-rank test $)$. 

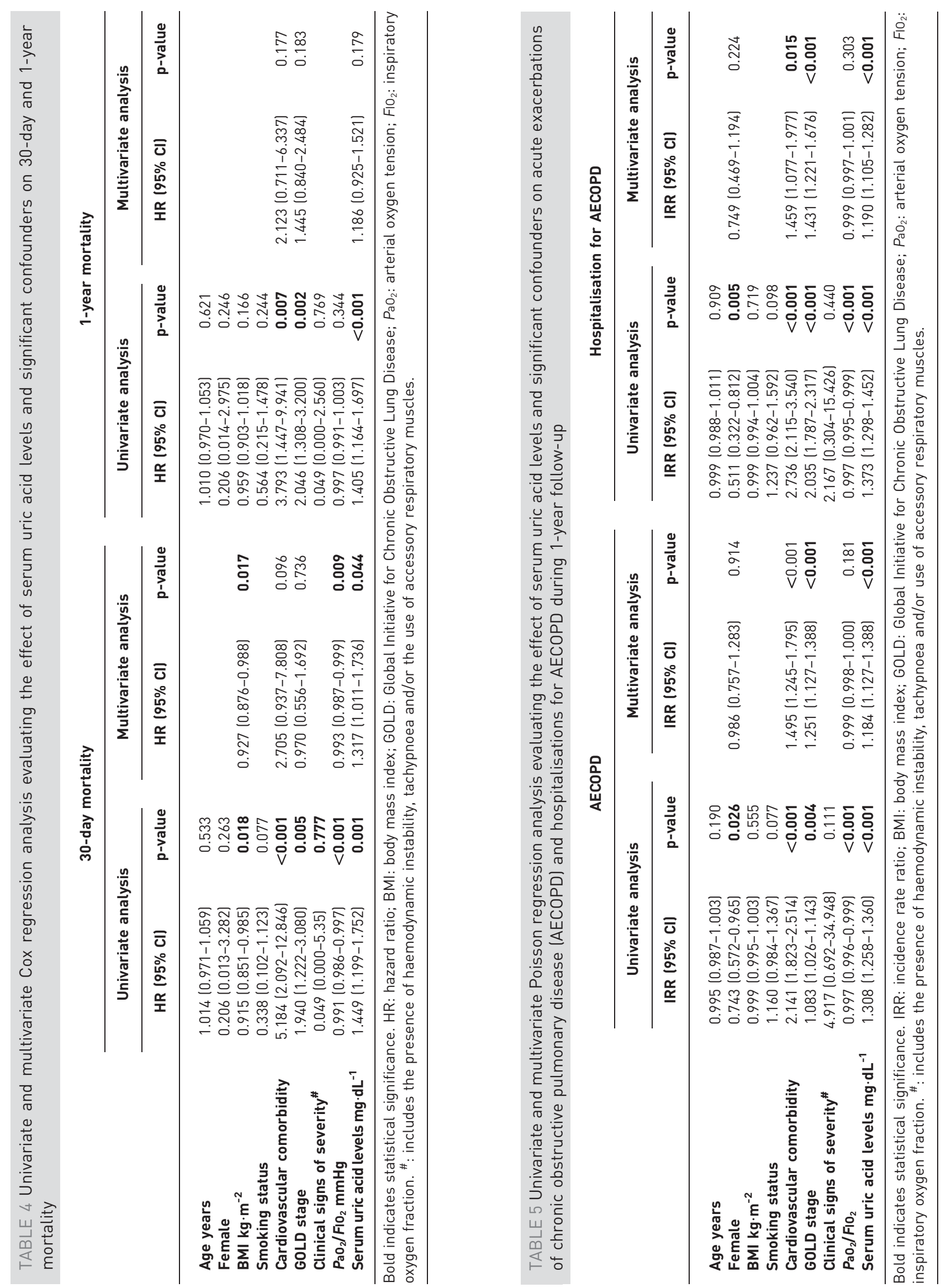

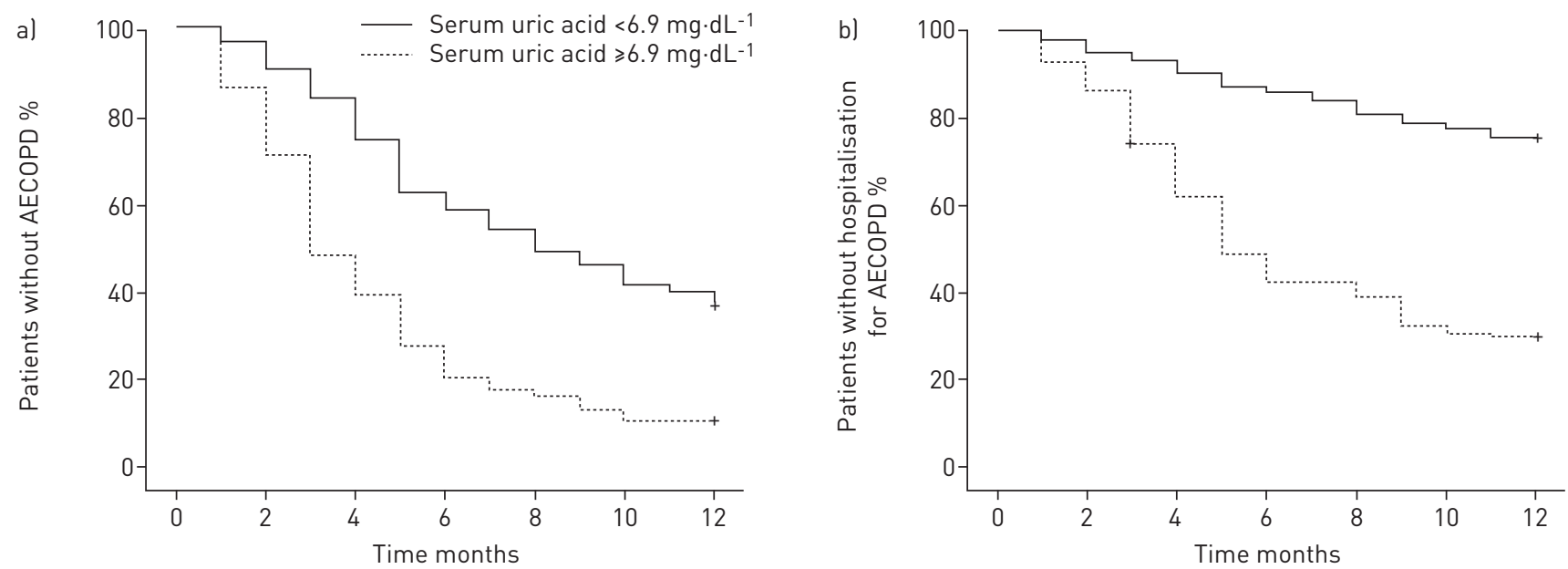

FIGURE 4 Kaplan-Meier curves evaluating a) the time to first acute exacerbation of chronic obstructive pulmonary disease (AECOPD) and b) the time to first hospitalisation for AECOPD for patients with uric acid levels above $\left(\geqslant 6.9 \mathrm{mg} \cdot \mathrm{dL}^{-1}\right)$ and below $\left(<6.9 \mathrm{mg} \cdot \mathrm{dL}^{-1}\right)$ the median value ( $<<0.001$ for both comparisons by log-rank test).

30 days. Serum uric acid levels were higher in patients with more severe airflow limitation and in frequent exacerbators. To our knowledge, this is the first study that has assessed serum uric acid, a widely available and rapidly measured biomarker, as a predictor of clinically important outcomes in a prospective cohort of patients with COPD.

Several mechanisms may be involved in the presence of high uric acid levels in AECOPD. First, prolonged hypoxaemia that is further increased during AECOPD may result in increased pulmonary artery pressures leading to increased right ventricle afterload, which promotes purine degradation through increased xanthine oxidase activity [17]. The negative association of serum uric acid levels with the $\mathrm{PaO}_{2} / \mathrm{FIO}_{2}$ ratio in our cohort provides support for this mechanism. Secondly, a considerable proportion of patients with COPD have comorbid cardiovascular disease which may be related to elevated uric acid [18], further supported by the more common cardiovascular disease in patients with increased uric acid levels in our cohort. Thirdly, impaired pulmonary function reduces oxygen intake resulting in tissue hypoxia which is more prominent during AECOPD, and this may lead to increased circulating uric acid levels originating from both lung and peripheral tissue damage. Finally, COPD is characterised by systemic inflammation [19], and elevated uric acid levels have been associated with increased levels of inflammatory markers (e.g. CRP and interleukin-6) [20] that may be important in the outcomes of COPD patients [21, 22].

Previous studies have attempted to identify biomarkers that predict clinically relevant outcomes during hospitalisation for AECOPD. In a previous study from our group, the measurement of several serum biomarkers, including CRP, serum amyloid-A, tumour necrosis factor- $\alpha$, interleukin- 6 and fibrinogen, failed to identify patients with early or late recovery from hospitalisation for AECOPD [23]. In a Swiss study, STOLZ et al. [24] showed that plasma pro-adrenomedullin and pro-endothelin levels were associated with the length of hospitalisation, but only pro-adrenomedullin is a predictor of long-term survival. Additionally, in another study from the same group, CRP and pro-calcitonin failed to predict short-term mortality, but increased levels of copeptin were associated with increased risk of death during hospitalisation [25]. The fact that high serum uric acid levels acted as an independent predictor of 30-day mortality suggest that this easy-to-obtain biomarker may be used to identify high-risk patients that require more intensive treatment. A recent study evaluated a combination of clinical parameters and biomarkers in order to identify independent predictors of outcome of hospitalisation for AECOPD and created the multicomponent DECAF (dyspnoea, eosinopenia, consolidation, acidaemia and atrial fibrillation) score [26]. Based on our data, serum uric acid may be evaluated as a candidate biomarker in a multicomponent score in future studies.

Uric acid levels have been previously associated with clinical and functional characteristics in patients with COPD in cross-sectional studies [9, 10, 27]. A Spanish study reported significant associations between serum uric acid/creatinine ratio and FEV1, forced vital capacity and MRC dyspnoea scale [10], but included a small sample of stable COPD subjects and no data about the outcomes of those patients. A large population-based spirometry study in Japan observed significant associations between serum uric acid levels and spirometry values in the general population [9], but study participants did not have physiciandiagnosed COPD. Finally, a study in 91 COPD outpatients receiving home oxygen therapy has suggested 
that serum uric acid/creatinine ratio is related to the survival of such patients [27], but the retrospective design and selection of a specific population may reduce the generalisability of the authors' conclusions for the general population of COPD patients. Although all the previously mentioned studies provide evidence that uric acid is increased in more severe disease, a population-based cross-sectional study by NicKs et al. [28] has shown reduced uric acid levels in patients with severe COPD. This discrepancy may be related to the differences in the origin of the populations studied, as well as to the fact that serum uric acid was measured at the time of hospitalisation for an AECOPD, in contrast to the stable condition of the patients studied by NICKS et al. [28]. In the present study, high serum uric acid levels were not an independent predictor of 1-year mortality in the multivariate Cox regression analysis, despite a trend in that direction. The different population and study designs may account for this discrepancy. However, the presence of a crude difference in mortality risk in patients with high uric acid levels in our cohort suggests a possible role for this biomarker as a predictor of long-term mortality which needs to be evaluated in larger studies.

We have shown that patients with increased uric acid levels presented more exacerbations in the 1-year follow-up, in contrast to data from GARCIA-PACHON et al. [10]; the prospective design of our study may account for this difference. This is further supported by the fact that frequent exacerbators in our study had increased serum uric acid levels at baseline compared to nonfrequent exacerbators. AECOPD are an important feature in the natural history of the disease and are associated with several risk factors, the most important being previous hospital admissions for AECOPD, comorbidities, more severe disease [29] and lower airway bacterial colonisation [30]. Recent data from the large ECLIPSE cohort suggest that the frequent-exacerbation phenotype is more common in more severe disease [13] and this may, in part, explain the associations between high serum uric acid levels with disease severity and exacerbation frequency in the 1-year follow-up in our cohort. However, the fact that uric acid levels remained an independent predictor of the risk for AECOPD and hospitalisations in the multivariate Cox regression analysis may suggest a possible role for this biomarker in the identification of exacerbation-prone patients.

Cardiovascular disease is associated with increased morbidity and mortality in COPD [18, 31]. Elevated uric acid levels were a strong independent marker of impaired prognosis in patients with cardiovascular disease $[32,33]$. A recent study showed that hyperuricaemia is associated with poor outcomes in heart failure patients without chronic kidney disease but not in those with chronic kidney disease, suggesting that hyperuricaemia may predict poor outcomes when it is related to increased xanthine oxidase activity, but not due to impaired renal excretion of uric acid [34]. The exclusion of patients with chronic renal failure in the present study suggests that the elevated serum uric acid levels may be associated with increased uric acid production in our population. Some of the patients with cardiovascular comorbidities were receiving medication known to influence uric acid levels, including aspirin or diuretics [35]. However, the fact that serum uric acid levels continued to be an independent predictor of 30-day mortality, even after adjustment for the presence of cardiovascular disease, further supports the possible role of uric acid as a clinically relevant biomarker in COPD. Another important observation is that the correlation of serum uric acid with $\mathrm{PaO}_{2} / \mathrm{FIO}_{2}$ is statistically significant but rather weak $\left(\mathrm{r}_{\mathrm{s}}=-0.188\right)$. This finding may well be attributed to the fact that $\mathrm{PaO}_{2}$ values at rest or even during an exacerbation do not necessarily reflect the whole spectrum of hypoxaemia in COPD patients. Dynamic hyperinflation and increased ventilation $\left(V^{\prime}\right) /$ perfusion $\left(Q^{\prime}\right)$ mismatching may lead to increased hypoxaemia during exercise, whereas decreased chemoreceptor sensitivity, diminished respiratory motor output and muscle contraction, and increased airflow resistance may be responsible for the pronounced nocturnal hypoxaemia in patients with COPD [36]. Cardiovascular comorbidities may be another confounder in this complex association.

In the evaluation of serum uric acid levels we need to take into account that this metabolite is the endproduct of purine degradation, which increases in a very sensitive but nonspecific way in several forms of tissue damage and inflammation, all of which are very dynamic processes in COPD patients [37, 38]. Uric acid levels are influenced by several factors including cardiovascular disease [6], food intake [39], alcohol consumption [40], renal dysfunction and genetic disorders of purine metabolism [6]. There are also genetic influences in the way COPD patients react to inflammation [41] which could have an important role in serum uric acid levels. In the present study we have excluded patients with chronic renal failure, and we performed all analyses based on the serum uric acid ratio, but we cannot exclude other possible confounders that may have influenced our results. However, despite these possible limitations, we believe that the data from our cohort provide evidence for a possible role of serum uric acid as a biomarker that is associated with disease severity and may identify patients with worse prognosis in hospitalised patients with AECOPD. Another important observation is that the correlation of serum uric acid with $\mathrm{PaO}_{2} / \mathrm{FIO}_{2}$ is statistically significant but rather weak $\left(\mathrm{r}_{\mathrm{s}}=-0.188\right)$. This finding may well be attributed to the fact that $\mathrm{PaO}_{2}$ values at rest or even during an exacerbation do not necessarily reflect the whole spectrum of hypoxaemia in COPD patients. Dynamic hyperinflation and increased $V^{\prime} / Q^{\prime}$ mismatching may lead to increased hypoxaemia during exercise, whereas decreased chemoreceptor sensitivity, diminished respiratory motor output and 
muscle contraction, and increased airflow resistance may be responsible for the pronounced nocturnal hypoxaemia in patients with COPD.

The present study presents some limitations. First, we excluded 17 patients from the analysis who were lost to follow-up early, immediately after their discharge from the hospital, due to the inability to establish contact with them afterwards. We decided to exclude them since this was an exploratory noninterventional study evaluating the possible role of serum uric acid as a predictor of clinically relevant outcomes which we wanted to capture accurately. Moreover, the overall demographics of those patients did not differ from the patients included in the analysis (15 (88.2\%) male, median age 73 years, $24 \%$ current smokers, median FEV1 $42 \%$ pred). Therefore, we believe that their exclusion has not significantly affected our results. Secondly, we do not have sufficient data about the previous history of AECOPD in our population. However, we believe that the prospectively collected data are more appropriate in order to characterise our patients as frequent exacerbators in the year of follow-up. Thirdly, our study design, involving monthly follow-up by telephone interview, may have led to the under-reporting of some events (AECOPD and/or hospitalisations) during follow-up. However, study investigators have made every effort to collect quality data, using hospital and/or patient records where necessary. Finally, for the same reason, we were not able to collect high-quality data on cause-specific mortality. However, the meticulous follow-up of our patients has accurately evaluated the time to death in all our deceased patients, therefore providing robust data regarding the time to death and all-cause mortality.

In conclusion, in the present study we have shown that serum uric acid levels on admission for an AECOPD are associated with increased 30-day mortality and increased risk of AECOPD and hospitalisation for acute exacerbations of COPD in 1 year. Our results, combined with the fact that serum uric acid is a widely and rapidly available, easy to interpret, low-cost biomarker, suggests a possible role for serum uric acid in the identification of COPD patients at increased risk of adverse outcomes that may need early intensive management.

\section{References}

Vestbo J, Hurd SS, Agustí AG, et al. Global strategy for the diagnosis, management and prevention of chronic obstructive pulmonary disease, GOLD executive summary. Am J Respir Crit Care Med 2013; 187: 347-365.

Soler-Cataluña JJ, Martínez-García MA, Román Sánchez P, et al. Severe acute exacerbations and mortality in patients with chronic obstructive pulmonary disease. Thorax 2005; 60: 925-931.

Fox IH. Metabolic basis for disorders of purine nucleotide degradation. Metabolism 1981; 30: 616-634.

Elsayed NM, Nakashima JM, Postlethwait EM. Measurement of uric acid as a marker of oxygen tension in the lung. Arch Biochem Biophys 1993; 302: 228-232.

Ruggiero C, Cherubini A, Ble A, et al. Uric acid and inflammatory markers. Eur Heart J 2006; 27: 1174-1181.

Feig DI, Kang DH, Johnson RJ. Uric acid and cardiovascular risk. N Engl J Med 2008; 359: 1811-1821.

Saito H, Nishimura M, Shibuya E, et al. Tissue hypoxia in sleep apnea syndrome assessed by uric acid and adenosine. Chest 2002; 122: 1686-1694.

8 Voelkel MA, Wynne KM, Badesch DB, et al. Hyperuricemia in severe pulmonary hypertension. Chest 2000; 117: 19-24.

9 Aida Y, Shibata Y, Osaka D, et al. The relationship between serum uric acid and spirometric values in participants in a health check: the Takahata study. Int J Med Sci 2011; 8: 470-478.

10 Garcia-Pachon E, Padilla-Navas I, Shum C. Serum uric acid to creatinine ratio in patients with chronic obstructive pulmonary disease. Lung 2007; 185: 21-24.

11 Papadopoulos A, Bartziokas K, Haniotou A, et al. Serum uric acid and uric acid/creatinine ratio in exacerbations of COPD. Eur Respir J 2011; 38: Suppl. 55, 157s.

12 Charlson ME, Pompei P, Ales KL, et al. A new method of classifying prognostic comorbidity in longitudinal studies: development and validation. J Chronic Dis 1987; 40: 373-383.

13 Hurst JR, Vestbo J, Anzueto A, et al. Susceptibility to exacerbation in chronic obstructive pulmonary disease. N Engl J Med 2010; 363: 1128-1138.

14 Høiseth AD, Neukamm A, Karlsson BD, et al. Elevated high-sensitivity cardiac troponin $\mathrm{T}$ is associated with increased mortality after acute exacerbation of chronic obstructive pulmonary disease. Thorax 2011; 66: 775-781.

15 McGhan R, Radcliff T, Fish R, et al. Predictors of rehospitalization and death after a severe exacerbation of COPD. Chest 2007; 132: 1748-1755.

16 Aaron SD, Fergusson D, Marks GB, et al. Counting, analysing and reporting exacerbations of COPD in randomised controlled trials. Thorax 2008; 63: 122-128.

17 Pascual-Figal DA, Hurtado-Martínez JA, Redondo B, et al. Hyperuricaemia and long-term outcome after hospital discharge in acute heart failure patients. Eur J Heart Fail 2007; 9: 518-524.

18 Hunninghake DB. Cardiovascular disease in chronic obstructive pulmonary disease. Proc Am Thorac Soc 2005; 2 : 44-49.

19 Fabbri LM, Rabe KF. From COPD to chronic systemic inflammatory syndrome? Lancet 2007; 370: 797-799.

20 Ruggiero C, Cherubini A, Miller E 3rd., et al. Usefulness of uric acid to predict changes in C-reactive protein and interleukin-6 in 3-year period in Italians aged 21 to 98 years. Am J Cardiol 2007; 100: 115-121.

21 Celli BR, Locantore N, Yates J, et al. Inflammatory biomarkers improve clinical prediction of mortality in chronic obstructive pulmonary disease. Am J Respir Crit Care Med 2012; 185: 1065-1072.

22 Dahl M, Vestbo J, Lange P, et al. C-reactive protein as a predictor of prognosis in chronic obstructive pulmonary disease. Am J Respir Crit Care Med 2007; 175: 250-255.

23 Koutsokera A, Kiropoulos TS, Nikoulis DJ, et al. Clinical, functional and biochemical changes during recovery from COPD exacerbations. Respir Med 2009; 103: 919-926. 
Stolz D, Christ-Crain M, Morgenthaler NG, et al. Plasma pro-adrenomedullin but not plasma pro-endothelin predicts survival in exacerbations of COPD. Chest 2008; 134: 263-272.

Stolz D, Christ-Crain M, Morgenthaler NG, et al. Copeptin, C-reactive protein, and procalcitonin as prognostic biomarkers in acute exacerbation of COPD. Chest 2007; 131: 1058-1067.

Steer J, Gibson J, Bourke SC. The DECAF Score: predicting hospital mortality in exacerbations of chronic obstructive pulmonary disease. Thorax 2012; 67: 970-976.

Sato N, Kurashima K, Ubukata M, et al. [Prognostic significance of serum uric acid in patients with chronic obstructive pulmonary disease receiving home oxygen therapy]. Nihon Kokyuki Gakkai Zasshi 2003; 41: 74-80.

Nicks ME, O’Brien MM, Bowler RP. Plasma antioxidants are associated with impaired lung function and COPD exacerbations in smokers. COPD 2011; 8: 264-269.

Miravitlles M, Guerrero T, Mayordomo C, et al. Factors associated with increased risk of exacerbation and hospital admission in a cohort of ambulatory COPD patients: a multiple logistic regression analysis. The EOLO Study Group. Respiration 2000; 67: 495-501.

Patel IS, Seemungal TA, Wilks M, et al. Relationship between bacterial colonisation and the frequency, character, and severity of COPD exacerbations. Thorax 2002; 57: 759-764.

1 Sin DD, Man SF. Why are patients with chronic obstructive pulmonary disease at increased risk of cardiovascular diseases? The potential role of systemic inflammation in chronic obstructive pulmonary disease. Circulation 2003; 107: 1514-1519.

Anker SD, Doehner W, Rauchhaus M, et al. Uric acid and survival in chronic heart failure: validation and application in metabolic, functional, and hemodynamic staging. Circulation 2003; 107: 1991-1997.

So A, Thorens B. Uric acid transport and disease. J Clin Invest 2010; 120: 1791-1799.

Filippatos GS, Ahmed MI, Gladden JD, et al. Hyperuricaemia, chronic kidney disease, and outcomes in heart failure: potential mechanistic insights from epidemiological data. Eur Heart J 2011; 32: 712-720.

Demartini FE. Hyperuricemia induced by drugs. Arthritis Rheum 1965; 8: 823-829.

Kent BD, Mitchell PD, McNicholas WT. Hypoxemia in patients with COPD: cause, effects, and disease progression. Int J Chron Obstruct Pulmon Dis 2011; 6: 199-208.

Gan WQ, Man SF, Senthilselvan A, et al. Association between chronic obstructive pulmonary disease and systemic inflammation: a systematic review and a meta-analysis. Thorax 2004; 59: 574-580.

8 Holme I, Aastveit AH, Hammar N, et al. Uric acid and risk of myocardial infarction, stroke and congestive heart failure in 417,734 men and women in the Apolipoprotein MOrtality RISk study (AMORIS). J Intern Med 2009; 266: $558-570$.

39 Choi HK, Liu S, Curhan G. Intake of purine-rich foods, protein, and dairy products and relationship to serum levels of uric acid: the Third National Health and Nutrition Examination Survey. Arthritis Rheum 2005; 52: 283-289.

Choi HK, Curhan G. Beer, liquor, and wine consumption and serum uric acid level: the Third National Health and Nutrition Examination Survey. Arthritis Rheum 2004; 51: 1023-1029.

1 Yanbaeva DG, Dentener MA, Creutzberg EC, et al. Systemic inflammation in COPD: is genetic susceptibility a key factor? COPD 2006; 3: 51-61. 